\title{
'Comment on the Paper "SLC20A2 and THAP1 deletion in familial basal ganglia calcification with dystonia" by Baker et al.'
}

\author{
João Oliveira
}

Received: 12 November 2013 / Accepted: 14 November 2013 /Published online: 22 November 2013

(C) Springer-Verlag Berlin Heidelberg 2013

Dear Editor,

I read with great interest the recent article by Baker et al., 2013 , reporting a patient with dystonia, bilateral brain calcifications, and carrying a remarkable heterozygous deletion $(\sim 500 \mathrm{~Kb})$, covering various interesting genes [1].

The authors highlight SLC20A2 and THAP1, both associated with familial brain calcification and dystonia, respectively. Curiously, patients with brain calcification can also exhibit dystonia. In this case, it is impossible to be sure if this deletion had an additive effect or not. However, I would like to call your attention to a small mistake in Figure 3. The gene DKK3 belongs to chromosome 11, not 8. DKK4 is located on chromosome 8 and should appear in this article.

A growing number of reports are identifying subjects with brain calcifications bearing mutations in the SLC20A2 gene. Besides the recent discovery of two other genes (PDGFB and $P D G F R B)$, most cases are linked to SLC20A2 which encodes a highly and widely expressed inorganic phosphate transporter [2].

Fifty percent of families tested so far were not associated with these three genes, reinforcing the fact that this condition is polygenic, clinically heterogeneous, and likely underdiagnosed. This highlights the need to increase clinical and neuroimaging awareness in order to increase detection.

\section{References}

1. Baker M, Strongosky AJ, Sanchez-Contreras MY, Yang S, Ferguson W, Calne DB, Calne S, Stoessl AJ, Allanson JE, Broderick DF, Hutton ML, Dickson DW, Ross OA, Wszolek ZK, Rademakers R. SLC20A2 and THAP1 deletion in familial basal ganglia calcification with dystonia. Neurogenetics. 2013 Oct 18. doi: 10.1007/s10048-013-0378-5 (in press)

2. Lemos RR, Ferreira JB, Keasey MP, Oliveira JR (2013) An update on primary familial brain calcification. Int Rev Neurobiol 110C:349-371. doi:10.1016/B978-0-12-410502-7.00015-6

J. Oliveira $(\bowtie)$

Federal University of Pernambuco, Recife, Pernambuco, Brazil

e-mail: joao.ricardo@ufpe.br 